\title{
The role of mediastinoscopy in the diagnosis of non-lung cancer diseases
}

\author{
This article was published in the following Dove Press journal: \\ Therapeutics and Clinical Risk Management \\ 27 July 2017 \\ Number of times this article has been viewed
}

\author{
Serdar Onat ${ }^{\prime}$ \\ Gungor Ates ${ }^{2}$ \\ Alper $\mathrm{Avcl}^{3}$ \\ Tekin Yıldız ${ }^{4}$ \\ Ali Birak' \\ Cihan Akgul Ozmen ${ }^{5}$ \\ Refik Ulku' \\ 'Department of Thoracic Surgery, \\ Faculty of Medicine, Dicle University, \\ ${ }^{2}$ Department of Chest Diseases, \\ Memorial Hospital, Diyarbakir, \\ ${ }^{3}$ Department of Thoracic Surgery, \\ Faculty of Medicine, Çukurova \\ University, Adana, ${ }^{4}$ Department \\ of Chest Diseases, Yuksek Ihtisas \\ Education and Research Hospital, \\ Bursa, ${ }^{5}$ Department of Radiology, \\ Faculty of Medicine, Dicle University, \\ Diyarbakir, Turkey
}

Background: Mediastinoscopy is a good method to evaluate mediastinal lesions. We sought to determine the current role of mediastinoscopy in the investigation of non-lung cancer patients with mediastinal lymphadenopathy.

Materials and methods: We retrospectively reviewed clinical parameters (age, gender, histological diagnosis, morbidity, mortality) of all patients without lung cancer who consecutively underwent mediastinoscopy in Hospital of Faculty of Medicine of Dicle University between June 2003 and December 2016.

Results: Two-hundred twenty nine patients without lung cancer who underwent mediastinoscopy for the pathological evaluation of mediastinum during the study period were included. There were 156 female (68\%) and 73 male (32\%) patients. Mean age was 52.6 years (range, 16 to 85 years). Mean operative time was 41 minutes (range, 25 to 90 minutes). Mean number of biopsies was 9.3 (range, 5 to 24). Totally, 45 patients (19.6\%) had previously undergone a nondiagnostic bronchoscopic biopsy such as transbronchial needle aspiration or endobronchial ultrasound-guided transbronchial needle aspiration. Mediastinoscopy was diagnostic for all patients. Diagnosis included sarcoidosis $(n=100)$, tuberculous lymphadenitis $(n=66)$, anthracosis lymphadenitis $(n=44)$, lymphoma $(n=11)$ metastatic carcinoma $(n=5)$, and Castleman's disease $(n=1)$; there was a diagnosis of silicosis in one patient and tymoma in one patient. Neither operative mortality nor major complication developed. The only minor complication was wound infection which was detected in three patients.

Conclusion: Although newer diagnostic modalities are being increasingly used to diagnose mediastinal diseases, mediastinoscopy continues to be a reliable method for the investigation of mediastinal lesions.

Keywords: mediastinoscopy, lymphadenopathy, granulomatous diseases, mediastinum, lymph node

\section{Introduction}

The standard method to diagnose and manage benign and malignant nodal disease is lymph node biopsy. Cervical mediastinoscopy (CM) is one of the methods to evaluate mediastinal lymph nodes. Mediastinoscopy is the most traditional method to evaluate mediastinal lesions ${ }^{1}$ and is still a current option to evaluate mediastinal lymph node lesions. ${ }^{2}$ In addition to allowing sampling of paratracheal and subcarinal lymph nodes, direct biopsy of mediastinal tumours is also possible with CM.

Over the years, new diagnostic tools such as transbronchial needle aspiration (TBNA), positron emission tomography/computed tomography (PET/CT), endoscopic ultrasound-guided fine needle aspiration (EUS-FNA), and endobronchial ultrasoundguided transbronchial needle aspiration (EBUS-TBNA) have been introduced for evaluating the mediastinum, especially in subjects with lung cancer. Many studies
Department of Thoracic Surgery, Faculty of Medicine, Dicle University, Kitilbil Street, Sur, 21280, Diyarbakir,Turkey Tel +904122488003

Email onatserdar2I@hotmail.com 
have evaluated the diagnostic value of mediastinoscopy in lung cancer diagnostic and staging algorithms. However, there is limited data about the diagnostic value of both CM and new diagnostic modalities in patients with noncancerous mediastinal lymphadenopathy. Herein, we reviewed our clinical experience of $\mathrm{CM}$ in patients with noncancerous mediastinal lymphadenopathy.

\section{Materials and methods}

Medical records of all patients without lung cancer who consecutively had undergone mediastinoscopy in our institution between June 2003 and December 2016 were retrospectively reviewed. Conventional mediastinoscopy has been replaced by video-assisted mediastinoscopy since May 2007. Mediastinoscopy was performed when enlarged (short axis $\geq 10 \mathrm{~mm}$ ) mediastinal lymph nodes or mediastinal masses were detected in the computed tomography (CT) image. Lymph node status was classified according to the international staging system reported by Mountain and Dresler. ${ }^{3}$ Patients with mediastinal lymph node enlargement due to non-lung cancer and with a previous bronchoscopic lavage, TBNA, or EBUS-TBNA were included. Patients who underwent mediastinoscopy with an aim to stage lung cancer were excluded. Most of the patients were suspected to have granulomatous disease such as sarcoidosis or tuberculosis. Standard fiberoptic bronchoscopy with bronchoalveolar lavage was performed before mediastinoscopy in all the cases suspicious of sarcoidosis. The diagnosis of sarcoidosis was made when the tissue sample revealed non-caseating granulomas along with compatible clinical features and exclusion of other causes for granulomatous inflammation (clinical history, follow-up, and a combination of negative tissue staining for acid-fast bacilli and negative respiratory mycobacterial cultures). As this was a retrospective study, approval from the local ethics committee was waived according to the regulations in our country.

All patients suspected to have lymphoma were examined for subcutaneous peripheral adenopathy. Data recording time of surgery, lymph node sampled, count of biopsies, number of sampled nodal levels, pre- and postoperative morbidity and mortality, histopathological diagnosis, and length of hospital stay were collected. The follow-up was assumed to be every 6 months in cases diagnosed by CM. This study was conducted in accordance with the Helsinki Declaration.

Mediastinoscopy was carried out according to the standard techniques. ${ }^{1,4}$ We did not perform extended CM as described by Ginsberg, ${ }^{29}$ in any of the patients. CM was performed in all patients under general anesthesia with single-lumen tube tracheal intubation. We used a supine position with a roll under the shoulders to extend the cervical area. A small incision was made in the suprasternal notch. A dissection was performed in the middle line between the strap muscles. Then, the pretracheal fascia was opened using scissors and separated from the anterior part of the trachea by digital dissection. We reached lymph nodes under innominate artery after dissecting pretracheal fascia. Wherever possible, lymph nodes were totally resected, otherwise only biopsies were taken. At least two lymph nodes were sampled. In the presence of mediastinal mass that could not be differentiated from conglomerate lymphadenopathy, sufficient tissue was taken for biopsy from the mass.

Statistical analysis was done. Continuous variables were expressed as means and ranges, and frequencies were expressed as percentages (\%).

\section{Results}

Two-hundred twenty nine patients without lung cancer underwent $\mathrm{CM}$ for the pathological evaluation of mediastinum during the study period. We included 156 female (68\%) and 73 male (22\%) patients. Mean age of the patients was 52.6 years (range, 16 to 85 years). Mean operative time was 41 minutes (range, 25 to 90 minutes). Mean number of lymph node biopsies was 9.3 (range, 5 to 24). Five hundred and ten nodal stations were biopsied. Mean level of sampled nodal number was 2.2 (range, 2 to 3 ). The most frequent nodal level biopsied was the right lower paratracheal (station 4R, $n=210$ ), followed by the right upper paratracheal (station $2 R$, $n=190$ ), the left upper paratracheal (station $2 \mathrm{~L}, \mathrm{n}=40$ ), the subcarinal (station 7, $\mathrm{n}=40$ ), and the left lower paratracheal (station $4 \mathrm{~L}, \mathrm{n}=30$ ) lymph nodes. Thirty-five lymph nodes were resected totally (Table 1).

Diagnosis included sarcoidosis $(n=100)$, tuberculous lymphadenitis ( $\mathrm{n}=66)$, anthracosis lymphadenitis $(\mathrm{n}=44)$, lymphoma $(n=11)$, metastatic carcinoma $(n=5)$, and Castleman's

Table I Sampling parameters of the patients

\begin{tabular}{ll}
\hline $\begin{array}{l}\text { Mean (range) number of } \\
\text { sampled nodes }\end{array}$ & $9.3(5-24)$ \\
\hline Nodal stations ( $\mathrm{n}=5 \mathrm{I} 0)$ & $4 \mathrm{R}, \mathrm{n}=210$ \\
$2 \mathrm{R}, \mathrm{n}=190$ \\
$2 \mathrm{~L}, \mathrm{n}=40$ \\
$7, \mathrm{n}=40$ \\
$4 \mathrm{~L}, \mathrm{n}=30$ \\
\hline Sarcoidosis, I00 (43.6) \\
Tuberculous lymphadenitis, 66 (28.8) \\
Anthracosis lymphadenitis, 44 (I9.2) \\
Lymphoma, I (4.8) \\
Metastatic carcinoma, 5 (2.9) \\
Castleman's disease, I (0.4) \\
Silicosis: I (0.4) \\
Thymoma, I (0.4) \\
\hline
\end{tabular}


disease $(n=1)$; there was a diagnosis of silicosis in one patient and thymoma in one patient (Table 1). The pathologic diagnosis was also confirmed by clinical and radiological findings (CT) in all cases during the follow-up and treatment period.

Altogether, 45 patients (19.6\%) had previously undergone a nondiagnostic bronchoscopic biopsy such as TBNA or EBUS-TBNA.

Neither operative mortality nor major complications developed. The only minor complication was wound infection which was detected in three patients. No late complications were recorded. Most of our patients were from out of the city, and therefore we advocated a routine 24-hour hospital stay. Mean length of patient follow-up was 52 months (range, 4 to 80 months).

\section{Discussion}

Mediastinoscopy is a common procedure to diagnose mediastinal diseases. Since its introduction by Carlens in 1959 , it has become the gold standard by which all other methods are evaluated. ${ }^{1}$ Although mediastinoscopy is most commonly used in the staging of lung cancer, it is useful for the diagnosis of non-lung cancer mediastinal lymph node diseases. ${ }^{2}$ $\mathrm{CM}$ is recommended by the European Society of Thoracic Surgeons (ESTS) guidelines as the standard procedure for staging with sampling of node stations 2,4 , and $7 . .^{5}$ Inability to sample posterior region of subcarinal lymph nodes may be the disadvantage of this method. EBUS-TBNA is recommended by the American College of Chest Physicians (ACCP) as the first-line method to stage lung cancer. ${ }^{6}$ Bronchoscopic lavage, TBNA, or EBUS-TBNA was applied to $19.6 \%(n=45)$ of our cases. Because EBUS has been a relatively new technique and followed in our hospital from past few years, we did not have sufficient experience in performing EBUS. Centers conducting EBUS as a routine procedure for many years have reported high sensitivity and recommended EBUS as the first-line method. ${ }^{7}$ The excellent results of EBUS have been reported from same limited number of centers with high experience. ${ }^{7-10}$ It has been reported the learning curve of EBUS-TBNA is more than 120 even in experienced bronchoscopists. ${ }^{11}$ Despite absence of such a number of patients and experienced pathologist, EBUS/ TBNA is increasingly used. ${ }^{12}$

Mediastinoscopy established a perfect diagnostic accuracy of the method used in our study. This is consistent with the data found in other reports. ${ }^{2,13}$ Tuberculosis is a common disease in our country and is responsible for most of the benign intrathoracic lymph node enlargements. ${ }^{14}$ Sixty-six of the 229 patients $(28.8 \%)$ in our study had a diagnosis of tuberculosis lymphadenopathy.

According to a statement issued by the American Thoracic Society, the European Respiratory Society, and the World Association of Sarcoidosis and Other Granulomatous Disorders, ${ }^{15}$ the diagnosis of sarcoidosis requires a compatible clinical picture, as well as demonstration of noncaseating granulomas without necrosis and exclusion of other probable diagnosis. Histological diagnosis is necessary before possible treatment and to rule out other diseases, such as lymph node metastases, lymphoma, or tuberculosis. ${ }^{16}$ In our study, 100 patients with sarcoidosis were successfully diagnosed by mediastinoscopy.

A diagnosis of sarcoidosis with conventional TBNA has been reported in $48 \%-82 \%$ of the cases, which is lower than that observed in the studies with EBUS-TBNA. ${ }^{17,18}$ Garwood et al demonstrated that EBUS-TBNA of mediastinal/ hilar lymph nodes may yield a granulomatous reaction in $85 \%$ of the patients suspicious of pulmonary sarcoidosis. ${ }^{18}$ The presence of non-caseating granulomas on cytological examination, however, was not sufficient to diagnose sarcoidosis. ${ }^{19}$ Other granulomatous diseases such as tuberculosis and histoplasmosis were not considered for a final diagnosis of sarcoidosis. However, granulomatous response to malignancy, especially lymphoma, has been described. ${ }^{20}$ It is highly important to obtain sufficient lymph node samples in order to differentiate sarcoidosis from other granulomatous diseases, especially in cases where tuberculosis is prevalent. Therefore, limited histological nodal sampling is not sufficient for an accurate diagnosis of sarcoidosis. ${ }^{21}$ Mycobacterial and fungal cultures should be performed to exclude the probability of infection in patients with any suspicion.

Excisional biopsy is recommended by the guidelines for diagnosis of lymphoma. ${ }^{22,23}$ The sensitivity of EBUS-TBNA technique may be as low as $22 \%$. Therefore, mediastinoscopy is proposed as the first-line method to evaluate lymphoproliferative diseases. ${ }^{24} \mathrm{We}$ diagnosed lymphoma in 11 cases in our study.

The rate of morbidity in our study is $1.3 \%$. This rate is similar to that reported in other studies. ${ }^{2,17,25,26}$ Perioperative bleeding, postoperative pneumothorax, wound infections, and temporary or permanent postoperative recurrent nerve paresis are probable complications. ${ }^{5,26}$ Absence of any type of morbidity in our population may be due to relatively low number of patients included in our study. The reason for the absence of temporary or permanent postoperative recurrent nerve paresis in our cases may be explained by avoidance of electrocautery or local anesthesia usage. ${ }^{27}$ The prevalence 
of major procedure-related bleeding was reported to be in the range of $0.1 \%$ to $0.4 \% .^{25}$ Absence of any cases having undergone previous radiotherapy may be the reason for not observing major bleeding in our population. In addition, five cases with malignant disorder had received systemic chemotherapy. Absence of repeat mediastinoscopy may also account for low mortality and morbidity in our cases. ${ }^{28}$

There were no cases of false-negative diagnoses in our study, which was reported to be up to $8.8 \%$ in other studies performed in patients with lung cancer. ${ }^{13}$ Because thoracotomy was not indicated in any of our patients, we were not able to present false-negative data. ${ }^{13}$

Mortality rates have been reported to be in a range of $0 \%$ to $0.1 \% .^{13,30}$ The mortality rate in our study was zero, which may be owing to a relatively low number of subjects. During the 13-year period, mediastinoscopy was found to be a safe procedure for the diagnosis of mediastinal diseases in our study.

Teaching mediastinoscopy has been difficult in the past with the conventional technique, but this has changed with the invention of video-mediastinoscopy. Exposure of anatomical landmarks such as the pulmonary artery, the vena azygos and vena cava, the left recurrent laryngeal nerve, and subcarinal lymph nodes is further facilitated by visualization on the video screen. ${ }^{13,30}$ This helps in teaching and training of the surgeon owing to improved visualization and easier dissection. Since 2007, we have been using video-mediastinoscopy in our institution.

Mediastinoscopy continues to have an important role in the sampling of masses and lymph nodes in mediastinum by providing a secure diagnosis to the patients with no lung cancer. Previous studies, however, reported mediastinoscopy to be more invasive and linked to a greater morbidity than TBNA. ${ }^{31}$ In contrast to these results, our study found that mediastinoscopy is a safe procedure with acceptable morbidity. In addition, lymph node sampling by mediastinoscopy has remained the "gold standard" approach for the differential diagnosis of mediastinal adenopathy. ${ }^{2}$

Despite prospective studies comparing EBUS-TBNA with mediastinoscopy to stage lung cancer, it will be difficult to perform prospective studies today because of the lack of data indicating thoracotomy in patients with noncancerous disease. ${ }^{7}$ Lacking of confirmation of EBUS-TBNA with mediastinoscopy as the gold standard method is reported to be a major limitation of studies with high diagnostic accuracy of EBUS-TBNA. ${ }^{32}$ Prospective studies that directly compare mediastinoscopy with new diagnostic methods such as EBUS and TBNA to diagnose noncancerous mediastinal adenopathy are warranted.
The limitations of this study are inclusion of single centered data, small sample size, and retrospective design.

\section{Conclusion}

Although newer diagnostic procedures are being employed to identify mediastinal diseases, mediastinoscopy is still a safe and effective method for investigating these diseases. Also, mediastinoscopy is currently a valid and valuable method and may be used where minimally invasive methods are unsuccessful. Mediastinoscopy should be regularly performed in thoracic surgery clinics to be able to be performed safely in the indicated patients in future.

\section{Disclosure}

The authors report no conflicts of interest in this work.

\section{References}

1. Carlens E. Mediastinoscopy: a method for inspection and tissue biopsy in the superior mediastinum. Dis Chest. 1959;36:343-349.

2. Wei B, Bryant AS, Minnich DJ, Cerfolio RJ. The safety and efficacy of mediastinoscopy when performed by general thoracic surgeon. Ann Thorac Surg. 2014;97(6):1878-1883.

3. Mountain CF, Dresler CM. Regional lymph node classification for lung cancer staging. Chest. 1997;111:1718-1723.

4. Gamliel Z. Mediastinal staging in non-small cell lung cancer. Surg Oncol Clin N Am. 2016;25(3):493-502.

5. De Leyn P, Lardinois D, Van Schil PE, et al. ESTS guidelines for preoperative lymph node staging for non-small cell lung cancer. Eur $J$ Cardiothorac Surg. 2007;32:1-8.

6. Silvestri GA, Gonzalez AV, Jantz MA, et al. Methods for staging non-small cell lung cancer: diagnosis and management of lung cancer, 3rd ed: American College of Chest Physicians evidence-based clinical practice guidelines. Chest. 2013;143(Suppl 5):e211S-e250S.

7. Um SW, Kim HK, Jung SH, et al. Endobronchial ultrasound versus mediastinoscopy for mediastinal nodal staging of non-small-cell lung cancer. J Thorac Oncol. 2015;10(2):331-337.

8. Yasufuku K, Pierre A, Darling G, et al. A prospective controlled trial of endobronchial ultrasound-guided transbronchial needle aspiration compared with mediastinoscopy for mediastinal lymph node staging of lung cancer. J Thorac Cardiovasc Surg. 2011;142:1393-1400.

9. Nakajima T, Yasufuku K, Saegusa F, et al. Rapid on-site cytologic evaluation during endobronchial ultrasound-guided transbronchial needle aspiration for nodal staging in patients with lung cancer. Ann Thorac Surg. 2013;95:1695-1699.

10. Herth FJ, Annema JT, Eberhardt R, et al. Endobronchial ultrasound with transbronchial needle aspiration for restaging the mediastinum in lung cancer. J Clin Oncol. 2008;26:3346-3350.

11. Fernández-Villar A, Leiro-Fernández V, Botana-Rial M, RepresasRepresas C, Núñez-Delgado M. The endobronchial ultrasound-guided transbronchial needle biopsy learning curve for mediastinal and hilar lymph node diagnosis. Chest. 2012;1411:278-279.

12. VanderLaan PA, Wang HH, Majid A, Folch E. Endobronchial ultrasound-guided transbronchial needle aspiration (EBUS-TBNA): an overview and update for the cytopathologist. Cancer Cytopathol. 2014;122(8):561-576.

13. Hammoud ZT, Anderson RC, Meyers BF, et al. The current role mediastinoscopy in the evaluation of thoracic disease. J Thorac Cardiovasc Surg. 1999;118:894-899.

14. Baran R, Tor M, Tahaoğlu K, et al. Intrathoracic tuberculous lymphadenopathy: clinical and bronchoscopic features in 17 adults without parenchymal lesions. Thorax. 1996;51:87-89. 
15. Hunninghake GW, Costabel U, Ando M, et al. ATS/ERS/WASOG statement on sarcoidosis: American Thoracic Society/European Respiratory Society/World Association of Sarcoidosis and other Granulomatous Disorders. Sarcoidosis Vasc Diffuse Lung Dis. 1999;16:149-173.

16. Hasan SB, Khan FW, Hashmi S, Ehtesham A, Magsi RA, Shahabuddin S. Cervical mediastinoscopy in the diagnosis of lymphadenopathy in South Asia. J Pak Med Assoc. 2016;66(Suppl 3)(10):S16-S18.

17. Karfis EA, Roustanis E, Beis J, Kakadellis J. Video-assisted cervical mediastinoscopy: our seven-year experience. Interact Cardiovasc Thorac Surg. 2008;7(6):1015-1018.

18. Garwood S, Judson MA, Silvestri G, Hoda R, Fraig M, Doelken P. Endobronchial ultrasound for the Diagnosis of Pulmonary Sarcoidosis. Chest. 2007;132:1298-1304.

19. Statement on sarcoidosis. Joint Statement of the American Thoracic Society (ATS), the European Respiratory Society (ERS) and the World Association of Sarcoidosis and Other Granulomatous Disorders (WASOG) adopted by the ATS Board of Directors and by the ERS Executive Committee. Am J Respir Crit Care Med. 1999;160:736-755.

20. Daly PA, O'Briain DS, Robinson I, Guckian M, Prichard JS. Hodgkin's disease with a granulomatous pulmonary presentation mimicking sarcoidosis. Thorax. 1988;43:407-409.

21. Baughman RP, Iannuzzi MC. Diagnosis of sarcoidosis: when is a peek good enough? Chest. 2000;117:931-932.

22. Dreyling M, Ghielmini M, Marcus R, Salles G, Vitolo U, Ladetto M; ESMO Guidelines Working Group. Newly diagnosed and relapsed follicular lymphoma: ESMO Clinical Practice Guidelines for diagnosis, treatment and follow-up. Ann Oncol. 2014;25(Suppl 3):iii76-iii82.
23. Du Rand IA, Barber PV, Goldring J, et al; British Thoracic Society Interventional Bronchoscopy Guideline Group. British Thoracic Society guideline for advanced diagnostic and therapeutic flexible bronchoscopy in adults. Thorax. 2011;66(Suppl 3):iii1-iii21.

24. Iqbal S, DePew ZS, Kurtin PJ, et al. Endobronchial ultrasound and lymphoproliferative disorders: a retrospective study. Ann Thorac Surg. 2012;94:1830-1834.

25. Lemaire A, Nikolic I, Petersen T, et al. Nine-year single center experience with cervical mediastinoscopy: complications and false negative rate. Ann Thorac Surg. 2006;82:1185-1190.

26. Goldstraw P. Mediastinal exploration by mediastinoscopy and mediastinotomy. Br J Dis Chest. 1988;82:111-118.

27. Velez-Cubian FO, Toosi K, Glover J, Pancholy B, Hong E. Aphonia after mediastinoscopy. Ann Thorac Surg. 2017;103(6):e549-e550.

28. Call S, Rami-Porta R, Obiols C, et al. Repeat mediastinoscopy in all its indications: experience with 96 patients and 101 procedures. Eur J Cardiothorac Surg. 2011;39(6):1022-1027.

29. Ginsberg RJ. Evaluation of the mediastinum by invasive techniques. Surg Clin North Am. 1987;67:1025-1035.

30. Lerut T, De Leyn P, Coosemans W, et al. Cervical videomediastinoscopy. Thorac Surg Clin. 2010;20(2):195-206.

31. Hegde PV, Liberman M. Mediastinal Staging: Endosonographic ultrasound lymph node biopsy or mediastinoscopy. Thorac Surg Clin. 2016;26(3):243-249.

32. Gahlot T, Parakh U, Verma K, Bhalotra B, Jain N. Endobronchial ultrasound-guided transbronchial needle aspiration in diagnosing mediastinal lymphadenopathy. Lung India. 2017;34(3):241-246.
Therapeutics and Clinical Risk Management

\section{Publish your work in this journal}

Therapeutics and Clinical Risk Management is an international, peerreviewed journal of clinical therapeutics and risk management, focusing on concise rapid reporting of clinical studies in all therapeutic areas, outcomes, safety, and programs for the effective, safe, and sustained use of medicines. This journal is indexed on PubMed Central, CAS,

\section{Dovepress}

EMBase, Scopus and the Elsevier Bibliographic databases. The manuscript management system is completely online and includes a very quick and fair peer-review system, which is all easy to use. Visit http://www.dovepress.com/testimonials.php to read real quotes from published authors. 